\title{
Ultrasonic test results before and after high heat flux testing on W- monoblock mock-ups of EU-DEMO vertical target
}

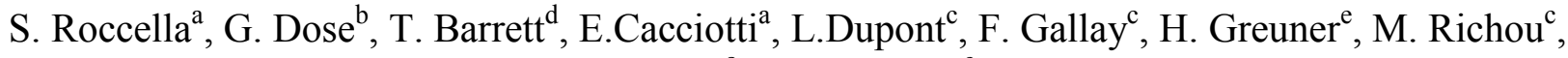 \\ E.Visca a and J-H. You \\ ${ }^{a}$ Department of Fusion and Technology for Nuclear Safety and Security, ENEA, Frascati, Italy \\ ${ }^{b}$ Università di Roma "Tor Vergata", Dipartimento di Ingegneria Industriale, Rome, Italy \\ ${ }^{c}$ CEA, IRFM, F-13108 Saint-Paul-Lez-Durance, France \\ ${ }^{d}$ CCFE, Culham Science Centre, Abingdon, Oxon, United Kingdom \\ ${ }^{e}$ Max-Planck-Institut für Plasmaphysik, Boltzmannstr. 2, Garching, Germany
}

\begin{abstract}
In the framework of the activity of target development of the European DEMO divertor, ENEA carried out an extensive ultrasonic testing campaign on more than 66 tungsten monoblock mock-ups. The EU-DEMO target development activity concerns primarily the comparison between the reference solution for the divertor targets (ITER-like) and two other concepts (Thermal Break Interlayer, Functionally Graded Interlayer), in which the interlayer between monoblocks and tube has been modified with the aim of increasing the component performance. In the same activity, many other aspects were also considered such as analyzing the influence of the tungsten monoblock supplier, interlayer thickness and interlayer manufacturing process (casting or diffusion bonding). Mock-ups were provided by ENEA (ITER-Like), CEA (Thin Graded Interlayer) and CCFE (Thermal Break Interlayer) and tested to thermal fatigue by high heat flux in the GLADIS facility in IPP - Garching center under DEMO relevant conditions. In this work the comparison between UT results obtained before and after the high heat flux test for the more significant mock-ups for phase 2 are carried out. After testing, mock-ups were cut in order to conduct post-mortem analyzes. Substantial agreement is found with UT results.
\end{abstract}

Keywords: Non-destructive examination, Ultrasonic testing, W monoblocks, DEMO divertor, Plasma-facing components, High heat flux testing

\section{Introduction}

The design of the divertor targets is one of the crucial points in the power exhaust issue. The life expectancy of a divertor made with the actual technology and design (ITER-like) under DEMO relevant loading conditions (DRLC), has been set to be two years. Technology readiness level of the ITER-like water-cooled target concept is substantially more progressed than other solutions (e.g. liquid metal, helium cooled divertor), and it is the principal strategy persecuted by EUROfusion Consortium [1]. Make improvements to the design and/or the materials starting from the ITER-like solution is the aim of the subproject 'Target Development', of the work package 'Divertor' (WPDIV).

In phase 1 of the "target development" subproject, several design concepts were being considered starting from the design studies up to the mock-ups fabrication and high-heat-flux testing (HHF). A second batch of mock-ups of all PFC concepts are produced and tested by HHF in phase 2, with an improved joining method, modified design or novel materials. The work presented here regards the results obtained in phase 2 based on the observations carried out through non-destructive examination by ultrasonic test (UT). The object treated here consists of the comparison between different designs of the interlayer proposed from the "target development" team.

Almost all of the mock-ups produced in the frame of this project have been tested in ENEA by UT technique.
In this work are analyzed the results of 5 mock-ups tested at HHF with the same thermal loads and hydraulic condition.

The UT equipment and data analysis software used is the one qualified to control the ITER divertor targets [3] and it has proved to be suitable also for the control of new mock-up designs during the phase 1 of this project [4]. The results obtained, in fact, were confirmed by the destructive investigations and a good accuracy in the dimensioning of the defects has been observed. This allows having a valid confidence on the results obtained in this second phase of the activity. The UT examination allows to evaluate both small defects in the material and modifications at the junction up to $0.2 \mathrm{~mm}$. The UT examination is carried out on the mock-ups before and after the HHF testing in the IPP GLADIS facility in order to check manufacture defects and to monitor modification under thermal fatigue testing

\section{Comparison between interlayer designs}

\section{$2.1 \quad$ Interlayer design}

The reference design of the plasma facing components for the DEMO divertor is similar to the ITER design (ITER-like design (IL)) where solid tungsten (W) blocks of the armor are cooled by water flowing into $\mathrm{CuCrZr}$ pipe passing through the $\mathrm{W}$ block. A pure copper compliance interlayer of $1 \mathrm{~mm}$ of thickness is placed between $\mathrm{W}$ blocks and the pipe to minimize the stress at the interface between materials with strong different thermal elongation. The dimensions 
of the $\mathrm{W}$ monoblock were optimized for DEMO environment conditions [5]. Fig. 1 shows the monoblock dimensions of the IL design. The IL mock-ups are manufactured in ENEA by hot radial pressing (HRP) process $\left(600^{\circ} \mathrm{C}, 50 \mathrm{MPa}, 2 \mathrm{~h}\right)$ [6].

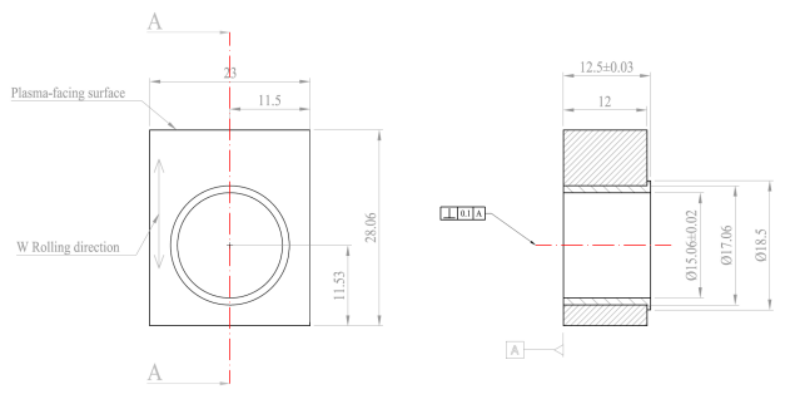

Fig.1. AT\&M and ALMT monoblocks geometry with $\mathrm{Cu}$ interlayer IL (1 $\mathrm{mm})$

Two alternative designs for the interlayer passed to phase2: functionally graded material (FGM) and thermal break (TB). For more details on the design and manufacturing processes of the mock-ups produced in the phase 2 can be found in [7] and [8] for the FGM concept and, in [9] and [10] for TB concept.

The FGM design consists of an interlayer made of $\mathrm{W} / \mathrm{Cu}$ pseudo-alloy with grading percentage of the two materials: the bore wall of the tungsten blocks is coated with $0.5 \mathrm{~mm}$ FGM layer by means of physical vapor deposition. The tungsten blocks are joined to the copper alloy pipe by hot isostatic pressing (HIP) technique. This design should allow substituting the ITER-like $\mathrm{Cu}$ interlayer with smaller thickness interlayer more resistant under neutron irradiation, but likewise able to mitigate stresses at the interface between different materials. Moreover, the smaller thickness brings lower temperatures of $\mathrm{W}$ armor and thus decreasing the recrystallized layer.

The idea of the TB concept is to mitigate the local concentration of heat flux at the cooling pipe by introducing a local thermal resistance from the HHF side, between $\mathrm{W}$ and pipe. The main advantages are the decrease of the pipe temperature and the increase of the margin from the critical heat flux $(\mathrm{CHF})$. A lower pipe temperature is useful to preserve the mechanical characteristics of the $\mathrm{CuCrZr}$ alloy under neutron irradiation and to decrease the erosion of the inner surface of the pipe due to the high water velocity (16 $\mathrm{m} / \mathrm{s})$.

The design proposed by CCFE consists of a thick interlayer $(2 \mathrm{~mm})$ with holes in the upper part. The increasing thickness brings about an increase on the maximum temperature achieved from the $\mathrm{W}$ monoblock loaded surface, thus increasing its recrystallization.

In addition, mock-ups with thin $(0.3$ and $0.1 \mathrm{~mm}) \mathrm{Cu}$ interlayer were manufactured by HRP with AT\&M monoblocks with HIPed interlayer, to found the minimum thickness needed to release the stresses at the interface.
With the aim to better compare the performance of the different interlayer, in the phase 2 , it was decided that the main dimensions of the $\mathrm{W}$ blocks were the same for all the concepts: $12 \mathrm{~mm}$ of thickness in the axial pipe direction, $23 \mathrm{~mm}$ in the perpendicular one and $8 \mathrm{~mm}$ of $\mathrm{W}$ in the plasma side to grant $5 \mathrm{~mm}$ of erosion (corresponding to 2 years of life at DRLC) without compromising the component. Also the material (CuCrZr alloy) and dimensions of the pipe (200 $\mathrm{mm}$ of axial length, $12 / 15 \mathrm{~mm}$ of diameter) are the same for all the mock-ups.

Even if the main dimensions of the blocks are the same for all the concepts, some differences are present. In the CCFE mock-ups the tungsten supplier is the Japanese company ALMT which provided the monoblocks with $\mathrm{Cu}$ interlayer performed by casting, melting of $\mathrm{Cu}$ in the hole. The tungsten monoblocks of the CEA samples come from the Chinese company AT\&M. With the aim of having a more rigorous comparison of the concepts, mock-ups ITER-like were manufactured by ENEA both with ALMT's monoblocks with $\mathrm{Cu}$ casting, and AT\&M's blocks. In this last case, the interlayer of $1 \mathrm{~mm}$ of pure $\mathrm{Cu}$ provided by AT\&M was obtained by HIP technique. The welding process between pipe and interlayer is: HRP for the ENEA mock-ups, HIP for CEA and vacuum brazing for CCFE mock-ups. During both the HIP and vacuum brazing process high temperature are reached which make a subsequent heat-treatment necessary for precipitation hardening of $\mathrm{CuCrZr}$ pipe.

Five mock-ups one for each interlayer concept (ILALMT, IL-AT\&M, TB, FGM and $300 \mu \mathrm{m}$ ) were tested to HHF thermal fatigue at the same load conditions and with the same cooling water hydraulic condition.

Table 1 reports the ID of these 5 mock-ups and summarizes the main characteristic of their interlayer.

Table 1. Main characteristic of the mock-up interlayer

\begin{tabular}{ccccc}
\hline ID & design & supplier & $\begin{array}{c}\text { interlayer } \\
\text { manuf. process }\end{array}$ & $\begin{array}{c}\text { Thickness } \\
{[\mathbf{m m}]}\end{array}$ \\
\hline ENEA28 & IL & ALMT & Casting & 1 \\
ENEA24 & IL & AT\&M & Hiping & 1 \\
ENEA19 & $300 \mu \mathrm{m}$ & AT\&M & Hiping & 0.3 \\
CEA12 & FGM & AT\&M & Hiping & 0.5 \\
CCFE8 & TB & ALMT & Casting & 2 \\
\hline
\end{tabular}

\subsection{HHF testing}

The HHF tests were performed at the neutral beam facility GLADIS at IPP Garching [11]. The HHF testing is conducted at DEMO relevant water conditions: 130 ${ }^{\circ} \mathrm{C}, 40$ bar and $16 \mathrm{~m} / \mathrm{s}$. The testing consists in screening tests heat flux was increased incrementally from 5 to 25 $\mathrm{MW} / \mathrm{m}^{2}$ where 5 loading cycles followed from the thermal fatigue test up to 500 cycles at $20 \mathrm{MWm}^{2}$. Each beam pulse lasts $10 \mathrm{~s}$, time interval in which the loaded sample reaches the thermal equilibrium. The surface temperature is monitoring by two IR pyrometers and high resolution $\mathrm{CCD}$ cameras. The measurement of the 
absolute value of the temperature can be challenging due to the low emissivity of W. However, the relative change in temperature during the course of cyclic loading is easier to assess and is used to monitor the integrity of the sample. Eventual hot spots (significant change in the intensity in an IR thermography) were been considered as the discriminant criterion: their absence indicates the test was passed.

\subsection{UT technique and result representation}

The UT method used is based on a pulse-echo water gap technique. The measurement equipment consist in a focused pulser/receiver probe, an echograph and an automatic handling system with two degrees of freedom, one translation and one rotation, both controlled by an integrated LabviewTMcode. The probe's piezoelectric crystals have a diameter of $3 \mathrm{~mm}$ and work at a nominal frequency of $15 \mathrm{MHz}$. More details can be found in [3]. The piezoelectric focalized probe is inserted in a special plastic probe holder designed to ensure that the distance between the probe and the interface to be analyzed is within its focal length. This probe holder is placed at the end of a rigid hollow shaft which allows to insert the probe inside the cooling tube of the mock-up to be analyzed. The mock-ups are fully immersed in water. The signal is acquired along the generatrix of the pipe ( $\mathrm{z}$ direction): the probe goes down inside the pipe and acquires the signals, then comes back and rotates by 1 degree (circumferential direction $\vartheta$ ). The test finishes after a rotation of $360^{\circ}$. The axial measurement step is $0.08 \mathrm{~mm}$. The UT system calibration for the IL components has been carried out in the frame of the full scale IVT prototype of the ITER divertor. The PFUs of the prototype has been manufactured and examined by UT in Special Technologies lab of R.C. ENEA in Frascati. A detailed discussion of the calibration method can be found in [12]. The capability to detect and size defects has been proven by scanning of an ad-hoc reference mock-up with artificial defects. Defects at the pipe/interlayer and interlayer/W interfaces have been detected up to a diameter of $0.5 \mathrm{~mm}$ with a sizing error in the order of the measurement step. Spherical defects with a diameter of $0.5 \mathrm{~mm}$ in the $\mathrm{CuCrZr}$ tube and in the copper interlayer have been detected, but their sizes are underestimated systematically due to the spherical geometry of the defect bottom. Non reference mock-ups are available for the TB and FGM concepts, however, the systematic comparison between UT results and subsequent, destructive investigations during phase 1 [4], confirmed the UT analysis results.

Different representations are used to show the results. The A-scan reports amplitude signal versus the distance traveled inside the material in every chosen point $(\mathrm{z}, \vartheta)$ (see Fig.13 below). Horizontal axis has been obtained setting a sound speed in the material, which allows to measure the distance traveled starting from the known time $(\mathrm{s}=\mathrm{v} \mathrm{t} / 2)$. The $\mathrm{Cu}$ sound speed of $4900 \mathrm{~m} / \mathrm{s}$ is set. The time origin is set where for the first times signal exceed a fixed threshold value (i.e. $0 \mathrm{~mm}$ corresponds to the pipe inner surface). The A-scan representation with abscissa in mm helps the localization of defect depth in the $\mathrm{Cu}$ up to the interface with a different material.

In the $\mathrm{C}$-scan representation the horizontal axis reports the axial displacement of the probe inside the pipe $(\mathrm{z}[\mathrm{mm}])$, while the vertical axis gives the rotation angle $\left(\vartheta\left[{ }^{\circ}\right]\right)$. The pixel color gives the maximum amplitude of the pressure signal inside of a chosen depth range. The zero in the axial direction is from the part of the water inlet during the HHF testing (high pressure (HP) side), while $\vartheta=0^{\circ}$ is from the opposite side of the HHF loaded surface; this mean the HHF zone is in the central part of the C-scan maps.

\section{$2.4 \quad$ Results}

All the mock-ups passed the HHF testing without showing relevant modification in the temperature of the loaded surface. Through the ultrasonic control it was possible to have useful indications on the state of the component before its failure and without destructive analysis.

Table 2 shows how mock-ups were chosen to compare the results. For example, CCFE8 is compared with ENEA28 because they share the same W supplier. In the same way, ENEA24 is chosen for the comparison between FGM and IL concepts, together with CEA12. ENEA28 and ENEA24 are also compared to see some difference in the performance of the joining with the pipe when the interlayer is manufactured by casting (ALMT) or HIPing (AT\&M). Finally, ENEA 24-19 and 26 with the same $\mathrm{W}$ supplier are used to analyze the interlayer thickness relevance.

Table 2. Choice of the mock-ups for each kind of comparison between interlayers.

\begin{tabular}{lcccc}
\hline ID & TB/IL & FGM/IL & $\begin{array}{c}\text { manuf. } \\
\text { process } \\
\text { (W Supplier) }\end{array}$ & Thickness \\
\hline ENEA 28 & $\mathrm{X}$ & $\mathrm{X}$ & $\mathrm{X}$ & $\mathrm{X}$ \\
ENEA 24 & & & & $\mathrm{X}$ \\
ENEA 19 & & $\mathrm{X}$ & & \\
CEA12 & $\mathrm{X}$ & & & \\
CCFE8 & $\mathrm{X}$ & & \\
\hline
\end{tabular}

\subsubsection{Thermal Break/ITER Like comparison}

The monoblock geometry with $\mathrm{TB}$ interlayer is shown in Fig.2.
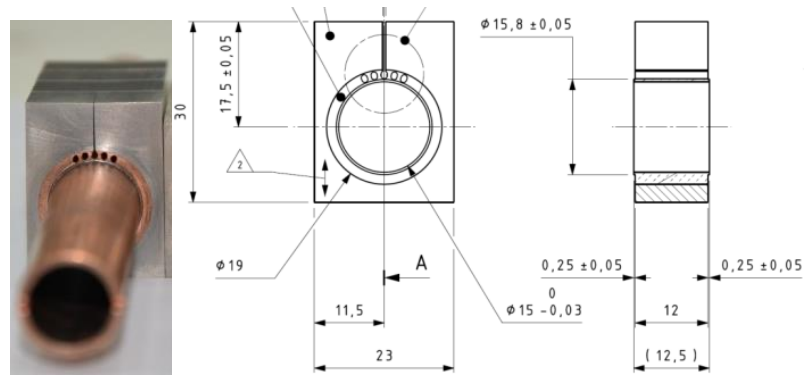

Fig.2. Monoblock geometry TB with structured interlayer.

External sizes of the monoblock are $12 \times 23 \times 30$ $\mathrm{mm}$. The monoblock has an interlayer of $2 \mathrm{~mm}$ of thickness made in $\mathrm{Cu}$ OFHC cast. Inside the interlayer 5 elongated holes were manufactured. 
Fig. 3 shows the internal surface of the pipe in the IL and TB mock-ups after the HHF testing. On the left the IL mockup shows vertical lines already present before the test: these lines are a modification of the internal surface typical of the HRP manufacturing process. In the figure the dashed box highlights the zone of the inner pipe from the side of the surface loaded during the HHF test. In this area can be see an indication not present before the HHF test: this type of signal indicates an erosion/corrosion of the surface due to the high water velocity and pipe temperature. On the right in the TB mock-up at $\mathrm{z}=30 \mathrm{~mm}$ and $\vartheta=200^{\circ} \div 320^{\circ}$ there can be see a scratch already present before the HHF test and new oblique signs. The inclination of these is in agreement with an imprinting of the swirl tape on the surface. In the CCFE mock-ups the swirl tape was made in stainless steel while in ENEA and CEA mock-ups it is made in copper. The greater mechanical strength of steel compared to copper and the expansion due to water temperature could be the causes of the clear sign left on the surface and present only in the CCFE mock-ups.

It is interesting to note the absence of the erosion/corrosion on the surface of the TB mock-up. This could depend on the lower pipe temperature due to a lower heat flux concentration. In this case, the results confirm the beneficial effect of the TB concept on the pipe integrity. However, the clear imprinting of the swirl tape testifies the perfect adhesion of the tape to the surface ensuring the correct flow of cooling water, while the ondulation of the surface in the ENEA mock-ups prevents tape adhesion and it could have a negative impact on the water flow circulation, accelerating the erosion process. Only one mock-up for each concept was tested with DEMO relevant hydraulic condition. The pipe corrosion is one of the main reasons of radioactive waste outside the machine; its cause deserves further investigation.

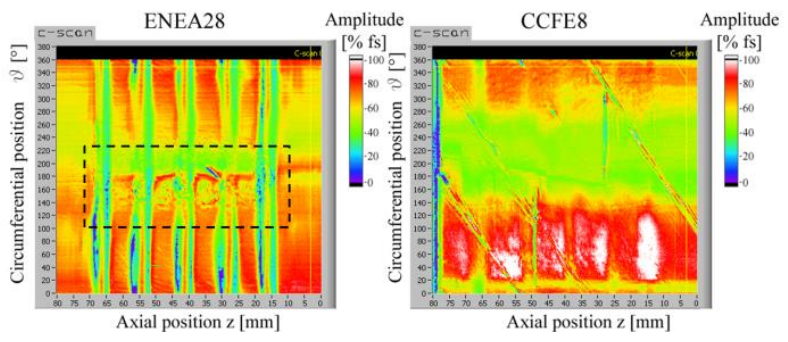

Fig.3. ENEA28 and CCFE8: C-scan at the interface between water and pipe

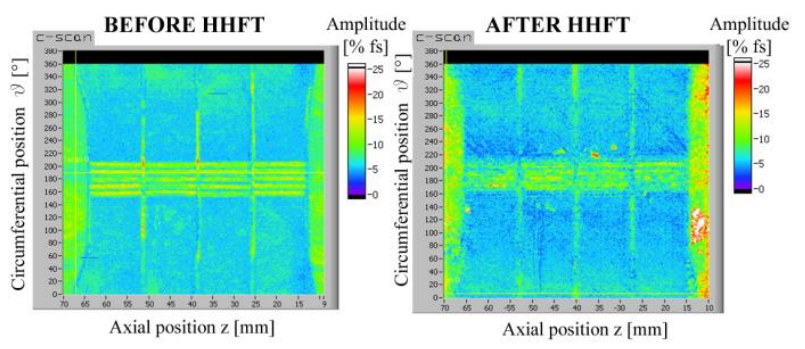

Fig.4. C-scans of the CCFE8 inside the interlayer before (on the left) and after (on the right) HHF testing. On the right can be see red/orange spots indicating porosity do not present before while the horizontal line coming from the holes appear to be fragmented.

To obtain the TB, the $\mathrm{Cu}$ interlayer thickness was increased in respect to the ITER-like design, thus achieving higher temperatures. A comparison between the condition of the interlayer before and after the fatigue test (see Fig.4) shows an increase of the porosity in the casting and large deformation of the 5 elongated holes.

The hole deformation has been confirmed by the subsequently metallographic analysis. The picture in Fig. 5 shows the HHF side of two monoblocks of the two mock-ups. On the right the degradation of the hole in the interlayer is evident. The different microstructure of the pipe, shown in Fig. 5, is a consequence of the different welding process. HRP preserves the microstructure of $\mathrm{CuCrZr}$ due to its lower temperature.

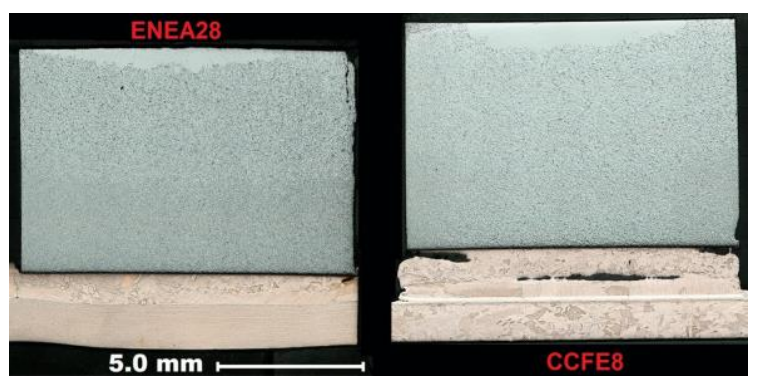

Fig.5. ENEA28 and CCFE8: metallography pictures after axial cutting, HHF side.

\subsubsection{Functionally Graded Material/ITER Like comparison}

The FGM interlayer was obtained varying the percentages of $\mathrm{Cu}$ and $\mathrm{W}$ inside the interlayer thickness. It was proposed FGM interlayer of different thickness and percentages of the constituting materials. The best results were obtained from the CEA12 mock-up (Fig.6), which is made with an interlayer of $0.5 \mathrm{~mm}$ thickness with the different percentages between the two external blocks $(25 \%$ vol.W/75\%vol.Cu) and the internal ones (50\%vol.W/50\%vol.Cu).

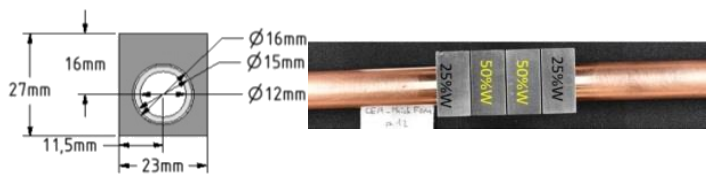

Fig.6. CEA12: monoblock dimensions and percentage of $\mathrm{W}$ inside the interlayer.

Fig.7 shows the pipe inner surface after testing of ENEA24 (on the left) and CEA12 (on the right): above, the UT results and below, photos after the axial cutting. From the UT results one can see that ENEA24 shows indications of erosion/corrosion on the surface, like ENEA28. Some indications of degradation of the pipe are evident also in the CEA mock-up, in particular between $z=30 \div 40 \mathrm{~mm}$, below the second monoblock starting from the HP side. The oblique green line is in agreement with the swirl tape position: the local greater amplitude of the signal in this zone (red color) indicates a local swelling of the pipe around the tape imprinting. The vertical line at $\mathrm{z}=42.5 \mathrm{~mm}$ is a machining defect 
already present: in fact, after the HIPing welding process the inner surface of the pipe must be machined to have the nominal diameter. Fig.7 below shows the parts of the mock-ups between $180^{\circ} \div 360^{\circ}$ related to the last two blocks. It is possible to see a deposit, probably of limestone, on the pipe surface of the ENEA mock-up, while the CEA one is clean. The pictures confirm the corrosion in both the cases even if more accentuated in the IL mock-up.

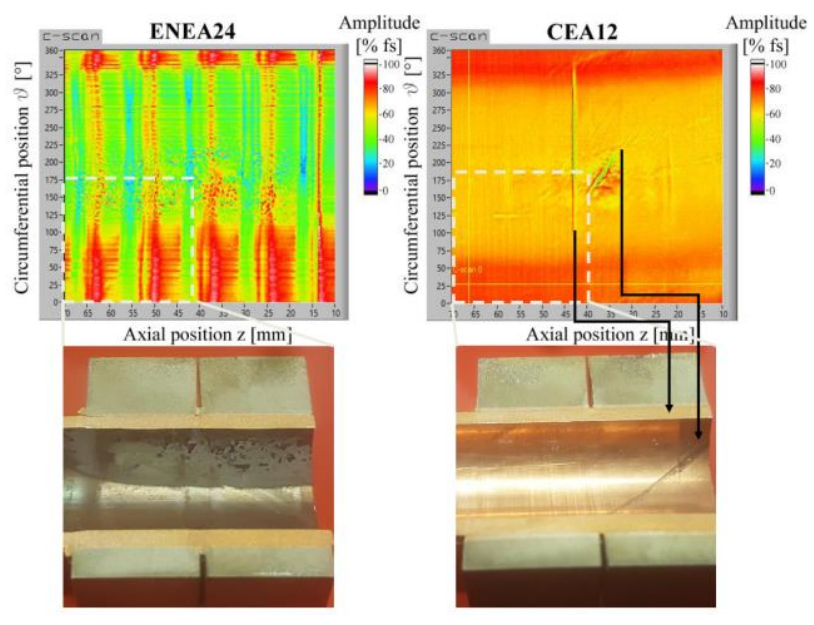

Fig.7. ENEA24 and CEA12: above C-scan of water/pipe interface; below pictures after axial cutting.

The good performances of the FGM mock-up are evident at the junction with the pipe. Fig. 8 on the left shows the interface between pipe and interlayer of the IL ENEA24. It is possible to see an increase in the debonding between the monoblocks: the initial gap of $0.5 \mathrm{~mm}$ between the blocks (the copper nose used to space the blocks does not weld with the tube during the HRP process) has become $1.4 \div 1.8 \mathrm{~mm}$ after 500 cycles at $20 \mathrm{MW} / \mathrm{m} 2$. Defects of these dimensions cannot be detected during the HHF testing by IR thermography. The same interface of CEA12 on the right, do not show any degradation. This could also be linked to the different joining process (HRP/HIP) and not only to the FGM interlayer. Manufacturing of a similar mock-up with $0.5 \mathrm{~mm}$ of interlayer in pure copper would be desirable for a more significant comparison.

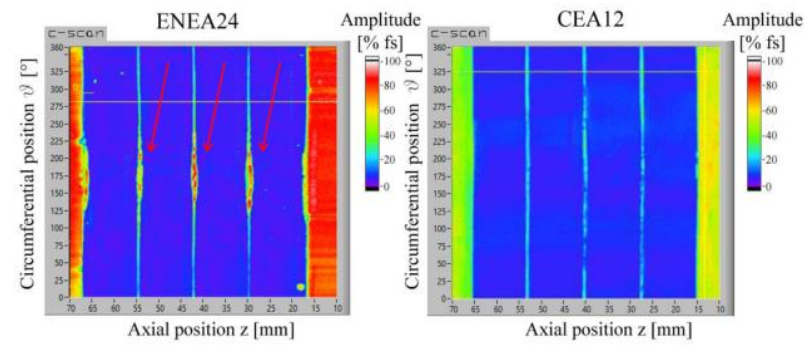

Fig. 8. ENEA24 and CEA12: C-scan of the interface pipe/interlayer; red arrows indicate debonding at the ends of the blocks.

Also the interlayer/W junction is free from defects (see Fig.9). This junction was critical during the FGM mock-up production process: extensive detachments were detected at this interface before the HHF testing. The CEA 12 was free from manufacturing defects and showed the best behavior under DRLC, but the manufacturing process of the FGM mock-ups led to high rate of failure. More details can be found in [7].

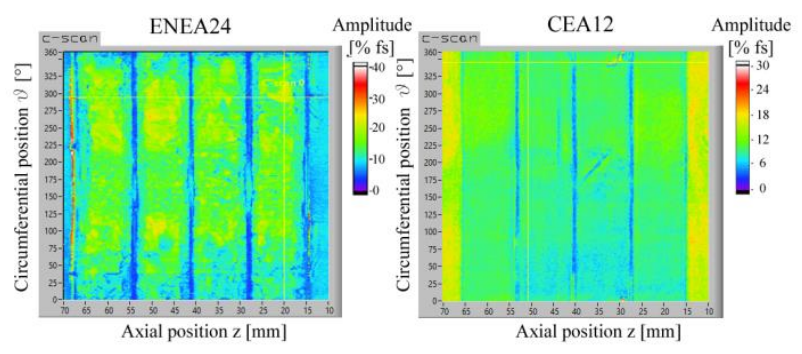

Fig.9. ENEA24 and CEA12: C-scan interface at interlayer/W interface.

\subsubsection{Interlayer thickness}

As said in the previous section IL mock-up (1 mm thickness interlayer) ENEA24 with AT\&M monoblocks survived the HHF testing with a very limited degradation of the interface between pipe and interlayer. Fig.10 and Fig. 11 show the UT result before and after the HHF test of the ENEA19 with 300 micron of interlayer. In Fig.10 $\mathrm{C}$-scan of the inner surface of the pipe shows the erosion/corrosion present in all the ENEA IL mock-ups. Fig.11 shows the interface pipe/interlayer: before circumferential debonding were detected at the ends of the mock-ups (maximum axial width $3 \mathrm{~mm}$ ). After testing, a strong degradation of the junction is evident. One can see an increase in the gaps between the blocks as in ENEA24, but in particular, large bands of debonding at $125^{\circ}$ and $215^{\circ}$ for all the four blocks.

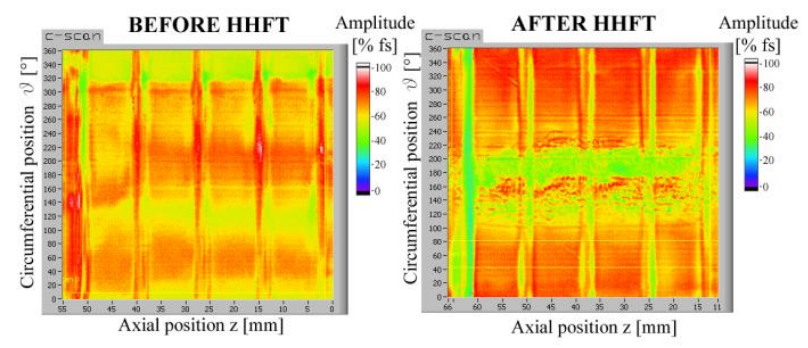

Fig.10. ENEA19 300 micron: interface water/pipe before (on the left) and after (on the right) HHF testing.

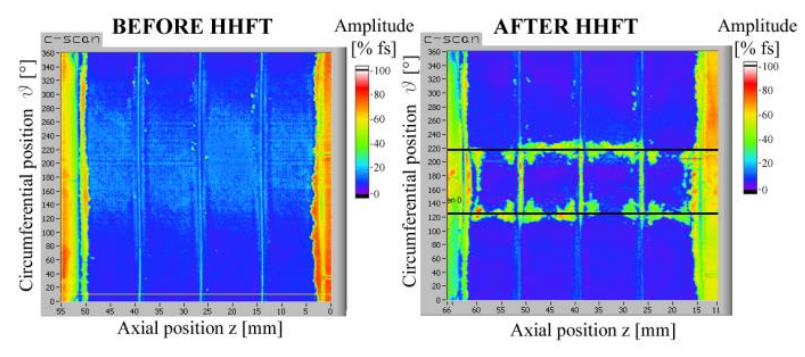

Fig.11. ENEA19 300 micron: interface pipe/interlayer before (on the left) and after (on the right) HHF testing.

A finite element calculation was carried out on a monoblock with the same geometry. A Chaboche model was used for $\mathrm{Cu}$ interlayer and $\mathrm{CuCrZr}$ pipe, while the $\mathrm{W}$ was considered elastic. The calculation is steady state, so here these results are reported only for some qualitative consideration and could be object of a future work. Fig. 12 reports the results in term of equivalent 
plastic strain: the maximum of the plastic deformation of the $\mathrm{Cu}$ copper at the interface with the pipe is at the same angular position as the detected defects. A same calculation for an IL monoblock (1 $\mathrm{mm}$ of interlayer) leads to an equivalent plastic deformation less than 0.012 $\mathrm{mm} / \mathrm{mm}$.
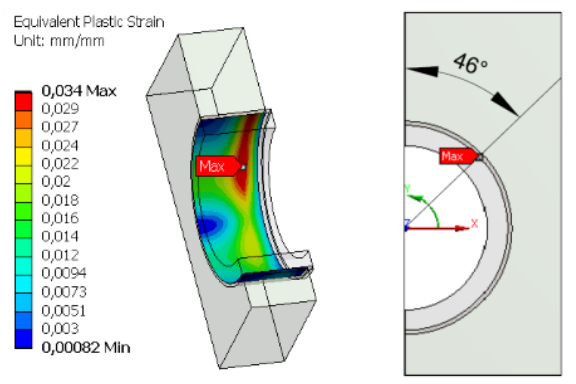

Fig.12. Numerical simulation: equivalent plastic strain.

\subsubsection{Manufacturing process and $W$ supplier}

Fig. 13 shows the results for ENEA24 and ENEA28: the only difference between the two mock-ups is in the monoblock supplier and, consequently, in the production process of the interlayer. In the mock-up 24, the interlayer is obtained through a solid-state diffusion welding, while in 28 through copper casting. The two Cscans in the figure are related to two different depths as indicated in the A-scan below. ENEA24, as mentioned, shows defects in the pipe/interlayer interface, while in ENEA28 defects are found near the interface with the W. The maximum axial width of these defects is $1.4 \mathrm{~mm}$ and it is at the ends of the blocks. The axial cutting validated the UT detection (see Fig.14 e Fig.15)

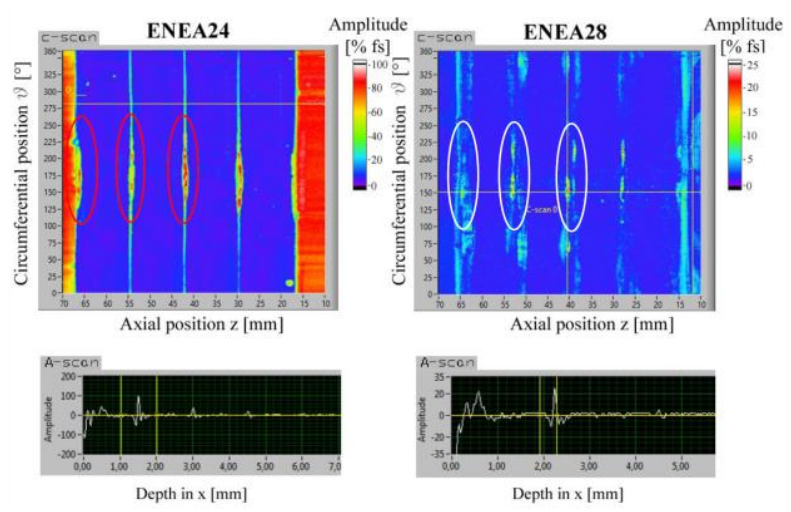

Fig.13. ENEA24 on the left: defects at the interface pipe/interlayer; ENEA28 on the right: defects near to the W.

Fig. 14 shows the debonding at the interface at the end of the blocks in ENEA24. In fig.15 the defects in ENEA28 are near the $\mathrm{W}$ and appear as an effect of thermal fatigue, while the HRP junction is not damaged.

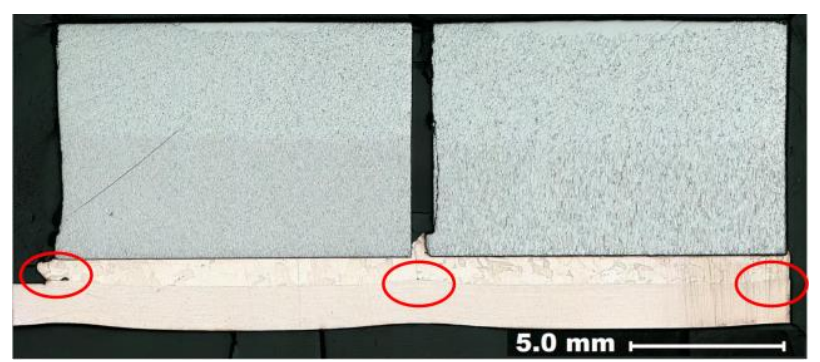

Fig.14. ENEA24: metallography image after axial cutting.

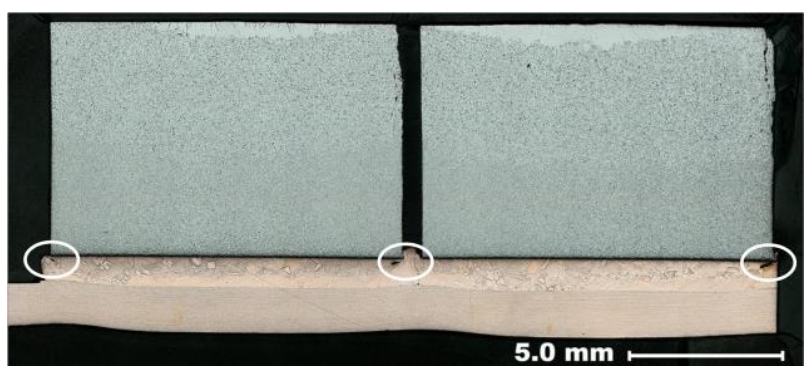

Fig.15. ENEA28: metallography image after axial cutting.

\section{Conclusions}

In the framework of the activity target development of the EU-DEMO divertor, ENEA carried out an extensive UT campaign. All concepts survived thermal fatigue testing without compromising the heat exhaust capability of the samples. In any case, some comparison can be carried out between designs.

FGM interlayer showed the best behavior under DEMO relevant load conditions. Not a single indication of degradation of the joining interfaces was found after 500 cycles at $20 \mathrm{MW} / \mathrm{m}^{2}$ with cooling water at $130^{\circ} \mathrm{C}$. Moreover, the amount of $\mathrm{Cu}$ in the interlayer is lower than in the IL: the copper is not a low activation material and is a priority to decrease as much as possible its quantity in the DEMO divertor. Improvements in the manufacturing process of PFC with FGM interlayer could be worth to pursue.

TB concept is able to preserve the pipe integrity and keeps away the CHF risk. UT was able to detect some effect due to thermal fatigue, namely porosity inside the interlayer and degradation of the spokes. The design should be reviewed to decrease the $\mathrm{Cu}$ quantity both for its degradation to high temperature and for its activation under neutron irradiation. The high erosion/corrosion found in the IL mock-ups suggests paying attention to the adhesion of the swirl tape with the pipe inner surface.

The HRP process validated its robustness also in DRLC and, metallographic analyzes showed, once again, its capability to preserve the lattice characteristics of the tube. The IL design has the best value for money and a greater number of cycles would be desirable to determine the application limits of this concept. Some differences were found in the defects in samples with a casted or a HIPed interlayer. As in both cases the heat exhaust capability was not compromised, it is hard to evaluate what is the best solution.

Finally, since the UTs are able to detect even small defects, they are a valid means to identify the beginning of the degradation of the junctions and provide a more global information of the sample than metallographic analysis, without missing any defect relevant to power exhaust. They could be used step by step during the HHF test to have useful information about performance degradation before the failure of the components.

\section{Acknowledgments}


This work has been carried out within the framework of the EUROfusion Consortium and has received funding from the Euratom research and training programme 2014-2018 and 2019-2020 under grant agreement No 633053. The views and opinions expressed herein do not necessarily reflect those of the European Commission.

\section{References}

[1] J.H. You, et al., Nuclear Materials and Energy 9 (2016) 171-176, http://dx.doi.org/10.1016/j.nme.2016.02.005

[2] J.H. You, et al., Nuclear Materials and Energy,16, 2018, 1-11 https://doi.org/10.1016/j.nme.2018.05.012

[3] S. Roccella, et al., Fusion Eng. and Des. 84 (2009),16391644.https://doi.org/10.1016/i.fusengdes.2008.12.096

[4] G. Dose, et al., Fusion Eng. and Des., 146, Part A, (2019), 870-873, https://doi.org/10.1016/j.fusengdes.2019.01.102

[5] M. Li*, J.-H. You., Fusion Eng. and Des.124(2017)468472, http://dx.doi.org/10.1016/j.fusengdes.2017.01.015

[6] E. Visca, et al., Fusion Eng. and Des. 136 B, 2018, 15931596, https://doi.org/10.1016/j.fusengdes.2018.05.064

[7] M. Richou, et al., Fusion Eng. and Des. 146 (2019) 858-861,https://doi.org/10.1016/j.fusengdes.2019.01.098

[8] Marc Missirlian, et al, Materials Science, 2019.

[9] F. Domptail, et al., Fusion Eng. and Des., 154, May 2020, https://doi.org/10.1016/j.fusengdes.2020.111497

[10] A. Lukenskas, et al., Fusion Eng. and Des.,146B, 2019, $1657-1660$ https://doi.org/10.1016/j.fusengdes.2019.03.010

[11] H. Greuner, et al., Physica Scripta, 2020, T171, https://doi.org/10.1088/1402-4896/ab3681

[12] S.Roccella , et al., ., Fusion Eng. and Des., 146, Part B, 2019, https://doi.org/10.1016/j.fusengdes.2019.03.189

[13] M. Richou, et al., Fusion Eng. and Des., 157, 2020, https://doi.org/10.1016/j.fusengdes.2020.111610. 